\title{
低氧诱导的 miR-210-3p 在非小细胞肺癌中预后价值的探讨
}

\section{Prognostic Value of Hypoxia-Induced miR-210-3p in Non-Small Cell Lung Cancer}

\author{
任杰 \\ Jie Ren
}

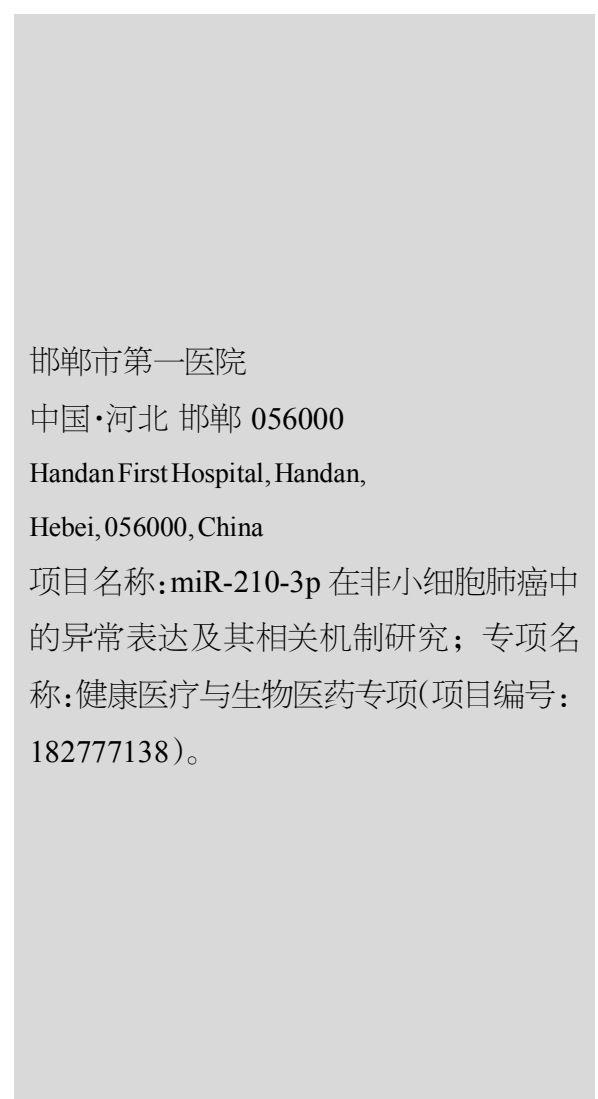

【摘 要】目的: 分析低氧诱导的 miR-210-3p 在非小细胞肺癌 (NSCLC) 中的预后价值。方 法: 选取我院行外科手术治疗的非小细胞肺癌患者 45 例为观察对象, 对组织标本中的 miR-210-3p 表达情况进行测定，分析 miR-210-3p 表达对于 NSCLC 预后评估作用。结果: miR-210-3p 高表达患者 25 例, 低表达 20 例。miR-210-3p 表达状态与患者复发情况之间存 在显著差异 $(P<0.05) ; \mathrm{miR}-210-3 \mathrm{p}$ 表达与年龄、肿瘤大小、分期等均不存在明显关联。结 论: miR-210-3p 在非小细胞肺癌中, 其高表达与患者的预后存在着显著关联, 可作为患者预 后判断的生物标志物。

【Abstract】Objective: To analyze the prognostic value of hypoxia-induced miR-210-3p in non-small cell lung cancer (NSCLC). Methods: 45 patients with non-small cell lung cancer were selected as observation objects, and the expression of miR-210-3p in tissue samples was determined to analyze the role of miR-210-3p expression in evaluating the prognosis of NSCLC. Results: There were 25 patients with high expression of miR-210-3p and 20 patients with low expression. There is a significant difference between the expression of miR-210-3p and the recurrence of patients $(P<0.05)$; The expression of miR-210-3p was not significantly correlated with age, tumor size and stage. Conclusion: The high expression of mir-210-3p in non-small cell lung cancer has a significant correlation with the prognosis of patients, which can be used as a biomarker for the prognosis of patients.

【关键词】低氧诱导; miR-210-3p; 非小细胞肺癌

【Keywords \hypoxia induction; miR-210-3p; non-small cell lung cancer

【DOI】10.36012/pmr.v2i2.1811

\section{1 引言}

在肺癌病例中, 非小细胞肺癌所占比例最高可达到 $80 \%$, 且其中多数患者在发现后已经发展到中晚期, 因此导致此疾 病患者的治疗难度较大, 生存率较低 ${ }^{[1]}$ 。因此, 有必要开展深入 的非小细胞肺癌临床诊断及预后评估等问题的研究 ${ }^{[2]}$ 。本次研 究中以 45 例非小细胞肺癌患者为观察对象, 对低氧诱导的 miR-210-3p 在非小细胞肺癌中的预后价值进行了分析。

\section{2 资料与方法}

\section{1 一般资料}

选取我院行外科手术治疗的非小细胞肺癌患者 45 例为

【作者简介】任杰(1975 年 ), 男, 回族, 河北大名人, 本科, 副主任, 从事肺癌研究。
观察对象。45 例患者中, 男性患者 25 例,女性患者 20 例; 最小 年龄患者 43 岁,最大年龄患者 76 岁。

\section{2 方法}

应用基于 raqMan 定量实施逆转录-聚合酶链反应对组织 样本 miRNA 表达水平进行测定。所有样品应用 RNU6B 为内 参实现数据标准化。经由与 30 个正常对照样品的中值对比核 算出各 NSCLC 样品 miR-210-3p 表达倍数变化情况。参考 miR-210-3p 表达水平将肺癌患者分组, 高出中位值纳入高表 达组, 反之则纳入低表达组。

\section{3 统计学处理}

本次研究中的所有数据均采用 SPSS17.0 统计软件进行处 理, 计量资料采用均数 \pm 标准差 $(\bar{x} \pm \mathrm{s})$ 表示, 以 $t$ 检验, 计数资 料采用百分比表示, 以卡方检验, $P<0.05$ 表示差异具有统计学 意义。 


\section{3 结果}

\section{1 肺癌患者 miR-210-3p 表达与临床特征}

本组患者中, miR-210-3p 高表达患者 25 例, 低表达 20 例。miR-210-3p 表达状态与患者复发情况之间存在显著差异 $(P<0.05) ; m i R-210-3 p$ 表达与年龄、肿瘤大小、分期等均不存 在明显关联(见表 1 )。

\section{表 1 肺癌患者 miR-210-3p 表达与临床特征}

\begin{tabular}{|c|c|c|c|c|c|}
\hline 病理因素 & & $\begin{array}{c}\text { 高表达组 } \\
(n=25)\end{array}$ & $\begin{array}{c}\text { 低表达组 } \\
(n=20)\end{array}$ & $t$ & $P$ \\
\hline \multirow[t]{2}{*}{ 年龄 } & $\leqslant 65$ 岁 & 6 & 7 & \multirow{2}{*}{1.253} & \multirow{2}{*}{0.203} \\
\hline & $>65$ 岁 & 19 & 13 & & \\
\hline \multirow[t]{2}{*}{ 肿瘤大小 } & $<30$ & 13 & 13 & \multirow{2}{*}{1.325} & \multirow{2}{*}{0.214} \\
\hline & $\geqslant 30$ & 12 & 7 & & \\
\hline \multirow[t]{2}{*}{ TNM 分期 } & $\mathrm{T} 1 、 \mathrm{~T} 2$ & 20 & 18 & \multirow{2}{*}{2.054} & \multirow{2}{*}{0.185} \\
\hline & $\mathrm{T} 3 、 \mathrm{~T} 4$ & 5 & 2 & & \\
\hline \multirow[t]{2}{*}{ 淋巴结 } & 无转移 & 11 & 11 & \multirow{2}{*}{1.241} & \multirow{2}{*}{0.206} \\
\hline & 转移 & 14 & 9 & & \\
\hline \multirow[t]{2}{*}{ 淋巴血管 } & 无浸润 & 14 & 15 & \multirow{2}{*}{1.262} & \multirow{2}{*}{0.235} \\
\hline & 浸润 & 11 & 5 & & \\
\hline \multirow[t]{2}{*}{ 复发 } & 有复发 & 14 & 6 & \multirow{2}{*}{4.265} & \multirow{2}{*}{0.038} \\
\hline & 无复发 & 11 & 14 & & \\
\hline
\end{tabular}

$3.2 \mathrm{miR}-210-3 p$ 表达与 NSCLC 无瘤生存率与

\section{总生存率}

经由 Kaplan-Meier 生存分析发现, miR-210-3p 高表达患 者与低表达患者对比, 无瘤生存率明显较低 $(P<0.05)$; miR210-3p 高表达患者与低表达患者 5 年无瘤生存率为 $35.00 \%$ 、 70.00\% ; miR-210-3p 高表达患者与低表达患者进行对比, 总 生存率明显较短 $(P<0.05), \mathrm{miR}-210-3 p$ 高表达患者总存活率 为 $45.00 \%$, 低表达患者的总存活率为 $75.00 \%$ 。

\section{$3.3 \mathrm{miR}-210-3 p$ 表达与肺腺癌与鳞状细胞癌}

\section{临床特征}

腺癌患者中 miR-210-3p 表达与病理分期、淋巴结转移、 疾病复发之间显著性相关。临床细胞癌患者中 miR-210-3p 表 达与患都临床特征无相关性(见表 2)。

\section{4 结语}

在非小细胞肺癌治疗方面，外科手术属于肺癌的优先选 择治疗手段, 也是核心治疗手段, 是唯一能够达到治愈效果的 治疗方案 ${ }^{[3,4]}$ 。外科手术治疗的目标是对肺癌原发病灶与转移 病灶进行完全切除, 以达到完全治愈的效果[5,6]。而对于患者的 预后评估还需要参考相关指标检测结果进行。 $m i R-210-3 p$ 高 水平表达患者的生存率要低于低表达患者, 且在腺癌中更为
表 $2 m i R-210-3 p$ 表达与肺腺癌、鳞状细胞癌临床特征关系

\begin{tabular}{|c|c|c|c|c|c|}
\hline 病理因素 & & $\begin{array}{c}\text { 高表达组 } \\
(n=23)\end{array}$ & $\begin{array}{c}\text { 低表达组 } \\
(n=22)\end{array}$ & $t$ & $P$ \\
\hline \multirow[t]{2}{*}{ 年龄 } & $\leqslant 65$ 岁 & 10 & 15 & \multirow{2}{*}{4.265} & \multirow{2}{*}{0.037} \\
\hline & $>65$ 岁 & 13 & 7 & & \\
\hline \multirow[t]{2}{*}{ 肿瘤大小 } & $<30$ & 11 & 10 & \multirow{2}{*}{1.253} & \multirow{2}{*}{0.25} \\
\hline & $\geqslant 30$ & 12 & 12 & & \\
\hline \multirow[t]{2}{*}{ TNM 分期 } & $\mathrm{T} 1 、 \mathrm{~T} 2$ & 10 & 10 & \multirow{2}{*}{1.024} & \multirow{2}{*}{0.274} \\
\hline & $\mathrm{T} 3 、 \mathrm{~T} 4$ & 13 & 12 & & \\
\hline \multirow[t]{2}{*}{ 淋巴结 } & 无转移 & 8 & 12 & \multirow{2}{*}{4.625} & \multirow{2}{*}{0.036} \\
\hline & 转移 & 15 & 10 & & \\
\hline \multirow[t]{2}{*}{ 淋巴血管 } & 无浸润 & 11 & 9 & \multirow{2}{*}{4.625} & \multirow{2}{*}{0.124} \\
\hline & 浸润 & 12 & 13 & & \\
\hline \multirow[t]{2}{*}{ 复发 } & 有复发 & 5 & 9 & \multirow{2}{*}{4.625} & \multirow{2}{*}{0.039} \\
\hline & 无复发 & 18 & 13 & & \\
\hline
\end{tabular}

显著。本次调查结果证实了 $\mathrm{miR}-210-3 \mathrm{p}$ 可作为肺腺癌的生物 标志物,但其高表达经由何种机制影响到患者预后还未清晰。 基于肺腺癌的实体肿瘤性质, 以及 $\mathrm{miR}-210-3 \mathrm{p}$ 会受到 HIFIa 直接调控, 认为可能与肺腺癌组织内细胞缺氧存在一定关 联。加之, 其属于缺氧条件中细胞内稳定性较强的 miRNA, 因 此可能会成为肺腺癌的标志物。而与腺癌进行对比, 其表达与 鳞癌病理参数并无明显关联, 代表着此项指标对于鳞癌患者 的预后影响还不能确定。

综上所述, miR-210-3p 在非小细胞肺癌中, 其高表达与 患者的预后存在着显著关联, 可作为患者预后判断的生物标 志物。

\section{参考文献}

[1]陈瑞琳,武润苗,吴桦,等.低氧诱导的 miR-210 在非小细胞肺癌 中预后价值的探讨[J].国际呼吸杂志,2019,39(2):101-106.

[2] 万鹏,黄庆,李昌林,等.术前 NLR、PLR 在非小细胞肺癌患者预 后中的价值[J].肿瘤预防与治疗, 2019,32(3):212-220.

[3]权琳,徐玲,陈文萍,等.外周血清蛋白和纤维蛋白原水平在晚期 非小细胞肺癌预后评估中的价值[J].肿瘤研究与临床,2019,31(12):828831.

[4]周晓亮,邓豪余,吴武林,等.18F-FDGPET/CT 显像及 VEGF 表 达在非小细胞肺癌预后评估中的价值[J].中国临床医学影像杂志,2019,30 (3):174-178.

[5]刘满庆,黄剑.肺癌组织不同核因子 E2 相关因子 2 及切除修复 交叉互补基因 1 水平在接受含铂化疗方案的晚期非小细胞肺癌患者 预后中的价值[J].安徽医药,2018,22(12):2369-2372.

[6]方申存,张映铭,张梅玲.中性粒细胞与淋巴细胞比率在评估晚期 非小细胞肺癌患者预后的价值 [J]. 临床肺科杂志,2018,23 (7): $1202-1204+1209$. 